\title{
A new stage of sentinel node navigation surgery in early gastric cancer
}

\author{
Takashi Fujimura $\cdot$ Sachio Fushida $\cdot$ Tomoya Tsukada $\cdot$ Jun Kinoshita $\cdot$ \\ Katsunobu Oyama $\cdot$ Tomoharu Miyashita $\cdot$ Hiroyuki Takamura • \\ Shinichi Kinami $\cdot$ Tetsuo Ohta
}

Received: 28 May 2014/ Accepted: 17 November 2014/Published online: 30 November 2014

(C) The International Gastric Cancer Association and The Japanese Gastric Cancer Association 2014

\begin{abstract}
Sentinel node (SN) navigation surgery is expected to realize organ- and function-preserving surgery with SN mapping, and has been applied in operations for breast cancer and melanoma. But there has been no definite evidence for the SN concept in gastric cancer. A prospective multicenter trial to confirm the SN concept for gastric cancer conducted by the Japan Society of Sentinel Node Navigation Surgery reported that the SN detection rate, sensitivity of positive SNs, and accuracy of nodal status are $97.5 \%$ (387/397), $93 \%$ (53/57), and $99 \%$ (383/ $387)$, respectively. A detailed analysis of the trial suggested that strictly the "lymphatic basin concept" rather than the "SN concept" was confirmed in early gastric cancer. The Japan Society of Sentinel Node Navigation Surgery started a new trial of function-preserving gastrectomy with lymphatic basin dissection (LBD) for early gastric cancer without metastasis in SNs on the basis of this promising outcome of the trial. It is supposed that LBD guarantees curability in SN navigation surgery for early gastric cancer. Full-thickness resection or endoscopic submucosal dissection in combination with laparoscopic LBD will soon be a new treatment option for early gastric cancer.
\end{abstract}

Keywords Sentinel node navigation surgery $\cdot$ Lymphatic basin - Selective lymphadenectomy · Function-preserving gastrectomy

T. Fujimura $(\bowtie) \cdot$ S. Fushida $\cdot$ T. Tsukada $\cdot$ J. Kinoshita

K. Oyama · T. Miyashita $\cdot$ H. Takamura $\cdot$ T. Ohta

Gastroenterologic Surgery, Kanazawa University,

13-1 Takaramachi, Kanazawa 920-8641, Japan

e-mail: tphuji@staff.kanazawa-u.ac.jp;

tphuji@tch.toyama.toyama.jp

S. Kinami

General and Digestive Surgery, Kanazawa Medical University,

Uchinada, Japan

\section{Introduction}

Patients receiving standard gastrectomy frequently have postgastrectomy syndrome, including dumping symptoms, early satiety, diarrhea, anemia, and bone disorder $[1,2]$. Because the incidence of early gastric cancer usually showing good prognosis has been increasing for the last two decades, postgastrectomy syndrome is a life-long problem for gastrectomized patients. Recently, some early gastric cancers with mucosal invasion have been treated with endoscopic therapies such as endoscopic mucosal resection (EMR) and endoscopic submucosal dissection (ESD) $[3,4]$. But most early gastric cancers need a standard operation including gastrectomy of more than two thirds of the stomach and lymphadenectomy of second-tier lymph nodes (D2 gastrectomy). Function-preserving gastrectomy with limited lymphadenectomy to improve the postoperative quality of life is recommended for patients with early gastric cancer for whom EMR or ESD is not applicable. But since 10-20\% of patients with early gastric cancer have lymph node metastasis, it is very difficult to reduce the extent of lymphadenectomy without recognizing the metastatic status of regional lymph nodes. Sentinel node (SN) navigation surgery (SNNS) is expected to diagnose lymph node metastasis intraoperatively and to realize function-preserving gastrectomy with selective lymphadenectomy [5, 6].

SNNS has been applied in surgery for breast cancer and melanoma. But there has been no definite evidence for the SN concept in early gastric cancer even though many articles have been published on a single-institute basis. Recently, a prospective multicenter trial conducted by the Japan Society of Sentinel Node Navigation Surgery demonstrated promising outcomes confirming the feasibility of SN biopsy for early gastric cancer [7]. On the basis of this 
result, several clinical studies for new treatments including function-preserving gastrectomy with individualized lymphadenectomy will be launched soon.

\section{Evidence of $\mathrm{SN}$ concept in gastric cancer}

It is a critical question whether the $\mathrm{SN}$ concept is applicable to gastric cancer, especially early gastric cancer. According to several studies from a single institute in not only Japan, Korea, and also other countries, it is reported that the detection rate, sensitivity, and accuracy are $55-100 \%, 40-100 \%$, and $70-100 \%$, respectively [8]. Ryu et al. [9] performed a meta-analysis of SN biopsy for gastric cancer, collecting 46 studies, which included 2,684 patients, published from 2001 to 2009. They reported that the sensitivity, detection rate, negative predictive value, and positive predictive value were $87.8,97.5,91.8$, and $38.0 \%$, respectively, warning that SN biopsy could not be clinically applicable for limited lymphadenectomy because of its low sensitivity and interstudy heterogeneity. Ryu et al. also showed that the number of harvested SNs (more than four) is the only factor to affect sensitivity. Another meta-analysis was reported, dealing with 38 articles and including 2,128 patients [10]. In this study, the SN identification rate, sensitivity, negative predictive value, and accuracy were 93.7, 76.9, 90.3, and $92.0 \%$, respectively. Early $\mathrm{T}$ stage, use of combined tracers, the submucosal tracer injection method, conventional open surgery, and pathological diagnosis by immunohistochemistry were associated with a higher $\mathrm{SN}$ identification rate and higher sensitivity.

Since it remains controversial whether the $\mathrm{SN}$ concept is valid or SNNS is feasible in gastric cancer, a large-scale prospective study is needed to confirm the SN concept. The Japan Society of Sentinel Node Navigation Surgery conducted a prospective multicenter trial to confirm the SN concept, optimal indications, and adequate methodology for gastric cancer [7]. Twelve institutes experiencing more than 30 cases of SN mapping participated in this trial. Gastric cancer patients assessed as having cT1N0M0 or cT2NOM0 disease with single primary lesions, smaller than $4 \mathrm{~cm}$ in diameter, and who had not received any specific treatments were considered as candidates for the study. Following biopsy of the identified SNs during an operation, mandatory D2 or modified D2 gastrectomy was performed according to the current gastric cancer treatment guidelines of the Japanese Gastric Cancer Association [3]. Finally, 397 of 433 registered patients were analyzed. The SN detection rate, sensitivity of positive SNs, and accuracy of nodal status were $97.5 \%$ (387/397), $93 \%$ (53/57), and $99 \%$ (383/387), respectively. Only four patients had falsenegative SN biopsy results, and pathological analysis revealed that three of the four patients had metastasis in a non-SN inside a lymphatic basin, an area containing stained lymph nodes and lymphatic vessels. One patient with metastasis in a non-SN outside a lymphatic basin had a primary tumor with a diameter of $4.9 \mathrm{~cm}$. This patient resulted in a case beyond the indication of this trial. These results suggested that strictly the "lymphatic basin concept" rather than the "SN concept" was confirmed in early gastric cancer. The Japan Society of Sentinel Node Navigation Surgery started a new trial of function-preserving gastrectomy with lymphatic basin dissection (LBD) for early gastric cancer on the basis of this promising outcome of the trial.

The Japan Clinical Oncology Group conducted another large multi-institutional trial (JCOG0302) [11]. The SN mapping in this study was performed by intraoperative serosal injection of indocyanine green (ICG), and sensitivity was estimated on the basis of intraoperative pathological examination. But this trial was discontinued owing to a high false-negative rate of $46 \%$ (13/28). The authors of the report analyzed a permanent pathological specimen of green nodes using additional paraffin sections, demonstrating that the false-negative rate was reduced to $14 \%$ (4/ 28). They supposed that intraoperative histological examination using only one section and a learning period with five patients for SN mapping in each institution caused the high false-negative rate. In the multicenter trial conducted by the Japan Society of Sentinel Node Navigation Surgery, 30 cases are required as the minimum initial learning phase according to the recommendation from the other multicenter report $[7,12,13]$.

\section{Practical aspect of SN mapping}

\section{Tracers}

Suitable tracers are necessary for detection of SNs. A dye or a radioisotope or both are used as tracers for SN mapping in gastric cancer. Dual-tracer mapping using both a dye and a radioisotope is recommended rather than singletracer mapping (dye or radioisotope) because the rate of detection of SNs in dual-tracer mapping is higher than that in single-tracer mapping, and only both of the tracers can detect metastases in SNs [7, 10].

Dyes such as sulfan blue (patent blue V), isosulfan blue (lymphazurin), and ICG are intraoperatively injected into gastric submucosa by endoscopy or into subserosa from the serosal side. They are very useful because of visibility, cheapness, safety, and an ability to stain not only lymph nodes but also lymphatic vessels. Dyes are, however, not preferable for patients with thick intraperitoneal adipose tissue or in the case of laparoscopic surgery. In general, 
blue dyes such as sulfan blue and isosulfan blue are more visible than a green dye such as ICG because background adipose tissue is colored yellow, a color complementary to blue. Thus, blue dyes were more prevalent than ICG in SN mapping, but recently ICG has replaced blue dyes because of allergic reaction and medical insurance in Japan.

But it is problematic that lower visibility of ICG contributes to a lower detection rate in $\mathrm{SN}$ mapping. To improve the rate of detection of SNs by ICG, Nimura et al. [14] developed a new fluorescence system to observe ICG absorption using infrared ray electronic endoscopy (Olympus Optical, Tokyo, Japan). They reported that the SN identification rate and sensitivity for infrared ray electronic endoscopy versus ICG alone were $99.5 \%$ versus $85.8 \%$, and $97.0 \%$ versus $48.4 \%$, respectively [15]. Moreover, another teype of fluorescence imaging using the ICG emission system was developed for SN mapping [16]. The photodynamic eye (PDE; Hamamatsu Photonics, Hamamatsu, Japan) is able to observe ICG fluorescence emitted by a light-emitting diode. The PDE clearly detects SNs compared with the usual method of staining with ICG itself. But a dark field in an operating room is necessary for detecting SNs in SN mapping by the PDE. Newly developed ICG fluorescence systems such as the HyperEye Medical System (Mizuho Medical, Tokyo, Japan) [17] and the D-light P system (Karl Storz, Tuttlingen, Germany) (Fig. 1) do not need room lights to be turned off for SN detection. The ICG concentration for detecting fluorescence should be as low as $0.05 \mathrm{mg} / \mathrm{ml}$. These systems make it possible for both mapping and retrieval of SNs to be performed under room light.

On the other hand, ${ }^{99 \mathrm{~m}} \mathrm{Tc}$-phytate or ${ }^{99 \mathrm{~m}} \mathrm{Tc}$-tin colloid is usually used as a tracer in gastric cancer. Since the half-life of ${ }^{99 \mathrm{~m}} \mathrm{Tc}$ is about $6 \mathrm{~h}$, the ${ }^{99 \mathrm{~m}} \mathrm{Tc}$ radioisotope tracer is injected 1 day before surgery, and the radioactivity of lymph nodes is detected intraoperatively using a gamma

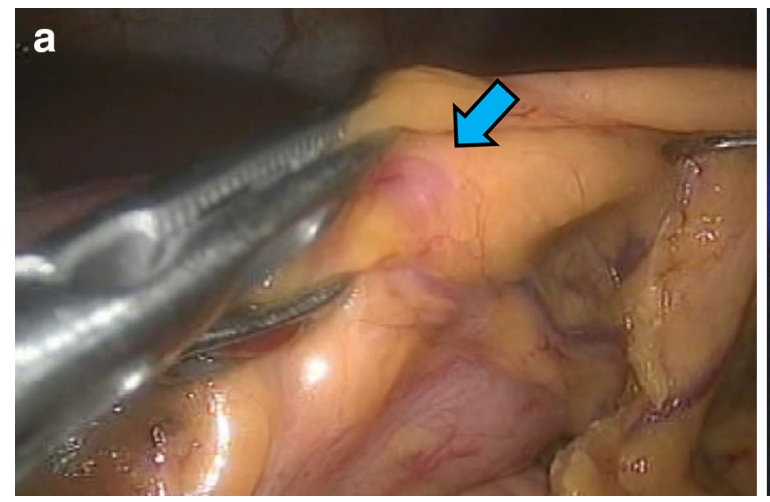

Fig. 1 A lymph node in the right gastroepiploic basin was detected with a fluorescence laparoscope with a near-infrared indocyanine green (ICG) emission system (D-light P system, Karl Storz, Tuttlingen, Germany). The regular mode (a) and the ICG emission mode probe. Preoperative lymphoscintigraphy is not routinely performed because its usefulness is very limited owing to a shine-through effect of the primary tumor and regional lymph nodes [8]. A great advantage of the radioisotope method is that it objectively measures the intensity of radioactivity and detects radioactivity even in thick intraperitoneal adipose tissue. Radioisotope tracers remain in lymph nodes for a relatively long time and so are preferable for laparoscopic surgery. The major drawbacks of radioisotope tracers are their high expense and the necessity of a radiation-controlled area.

\section{Injection of tracers}

Both submucosal and subserosal injections of tracer have been developed for SN mapping. Submucosal injection of the tracer using an endoscope is a standard procedure in the trial conducted by the Japan Society of Sentinel Node Navigation Surgery [6]. In our institute, a tracer is endoscopically injected into the submucosal layer at four points around a primary tumor. Then, $0.5 \mathrm{ml}$ of ${ }^{99 \mathrm{~m}} \mathrm{Tc}$ tin colloid or $0.2 \mathrm{ml}$ of $2 \%$ sulfan blue is injected at each point. No evidence of cancer was confirmed at these four points, where tiny clips are placed to identify the location [18].

Several researchers reported that there was no difference in the detection rate, mean number of SNs, and sensitivity of the SN biopsies between submucosal and subserosal injection $[19,20]$. But submucosal injection seems theoretical because lymph flows from the mucosal side to the serosal side and cancer cells move in this direction. On the other hand, subserosal administration is not suitable for preoperative injection of a radioisotope tracer or intraoperative injection with the laparoscopic approach. Since the inferiority of subserosal injection compared with submucosal injection in the rate of detection and sensitivity of SNs was reported in a meta-analysis [10], submucosal

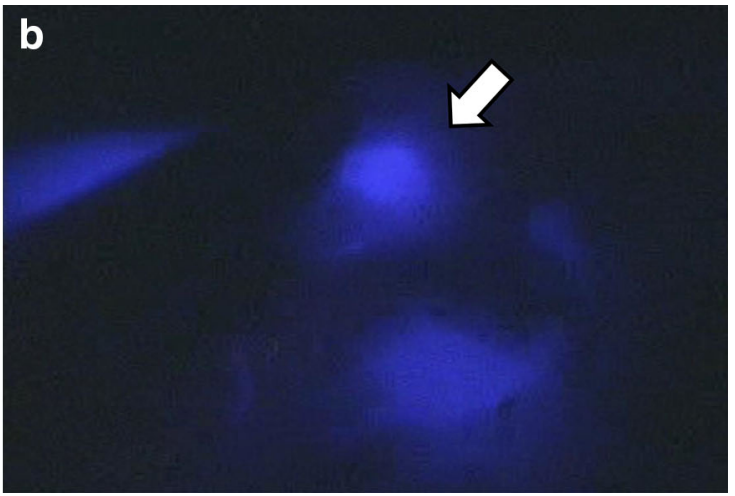

(b) of the D-light P system are instantly interchangeable using a foot switch. The lymph node observed with the regular mode (blue arrow) does not seem to be stained by ICG, whereas it is clearly identified as a sentinel node with the ICG emission mode (white arrow) 
injection is recommended for SN mapping in early gastric cancer.

\section{Retrieval of SNs}

Two methods to retrieve SNs have been reported: the pickup method and LBD (Fig. 2). The pick-up method is a very popular method in SNNS for breast cancer and melanoma. Only stained and/or hot nodes, which are regarded as SNs, are retrieved to examine the metastatic status by intraoperative pathological examination. LBD is a selective lymphadenectomy to dissect stained areas, so-called lymphatic basins, containing lymph nodes and lymphatic vessels stained with dye. Stained and/or hot nodes are retrieved on a back table just after the LBD and are submitted to intraoperative pathological examination. There are five lymphatic basins along the main arteries of the stomach: the left gastric artery, right gastric artery, left gastroepiploic artery, right gastroepiploic artery, and posterior gastric artery [21]. The type of surgery chosen depends on the tumor location and the number and location of the lymphatic basins.

According to our feasibility study of SN mapping, more than half of patients with metastases to stained or hot nodes also had metastases to nonstained or cold nodes. But these nonstained or cold nodes with metastases existed in the lymphatic basin including stained nodes, except in one case $[18,21]$. This patient had an advanced cancer with a large number of metastatic nodes; M, Gre, type 3, $3 \mathrm{~cm}$, pT2 (SS), pN1 (10/44) [21]. If metastases in stained or hot nodes cannot be detected by intraoperative biopsy in the case of the pick-up method, metastases in nonstained or cold nodes inside the lymphatic basin will be left. But LBD would succeed in dissecting metastatic lymph nodes in not only stained/hot nodes, but also in nonstained/cold nodes in the basin. This "lymphatic basin concept" was validated in the feasibility study conducted by the Japan Society of Sentinel Node Navigation Surgery [7].

From the results taken together, LBD might guarantee curability in SNNS for early gastric cancer, but the results of the new trial conducted by the Japan Society of Sentinel Node Navigation Surgery based on the LBD concept are still awaited to confirm the curability of function-preserving surgery. Kelder et al. [15] reported that the rate of lymph node metastasis was $50 \%$ with the pick-up method versus $92.3 \%$ with LBD, and nodal metastasis was always in the lymphatic basin. At this time, the pick-up method is not applicable to clinical practice of SN biopsy in gastric cancer owing to insecurity in oncological curability and complexity under laparoscopic surgery [22].

\section{Intraoperative diagnosis of SNs}

Intraoperative biopsy using frozen sections of SNs is the most important but complicated issue of SN mapping. Overlooking microscopic detection of metastatic cancer cells as well as macroscopic detection of SN nodes causes false negatives in the SN mapping. In particular, micrometastasis influences the false-negative rate in intraoperative pathological diagnosis. It was reported that $24 \%$ of patients with SN metastasis diagnosed by permanent sections could not be identified by frozen sections during the operation in the trial conducted by the Japan Society of Sentinel Node Navigation Surgery [7].

Different methods such as multistep level section, immunohistochemistry [23, 24], reverse transcription PCR (RT-PCR) [25-28], and the one-step nucleic acid amplification assay [29] have been developed to reduce the falsenegative rate by an additional diagnosis for micrometastasis in SNs. Micrometastases were detected in four of 35 patients $(11 \%)$, and six of 1,028 nodes $(0.58 \%)$ without metastasis diagnosed by permanent section, using a
Fig. 2 Methods to retrieve sentinel nodes. Only stained and/or hot nodes, which are regarded as sentinel nodes (asterisks), are retrieved to examine the metastatic status by intraoperative pathological examination in the pick-up method (a). Lymphatic basin dissection (b) is a selective lymphadenectomy to dissect stained areas, so-called lymphatic basins (dotted region), containing lymph nodes and lymphatic vessels stained with dye. $R t$ right

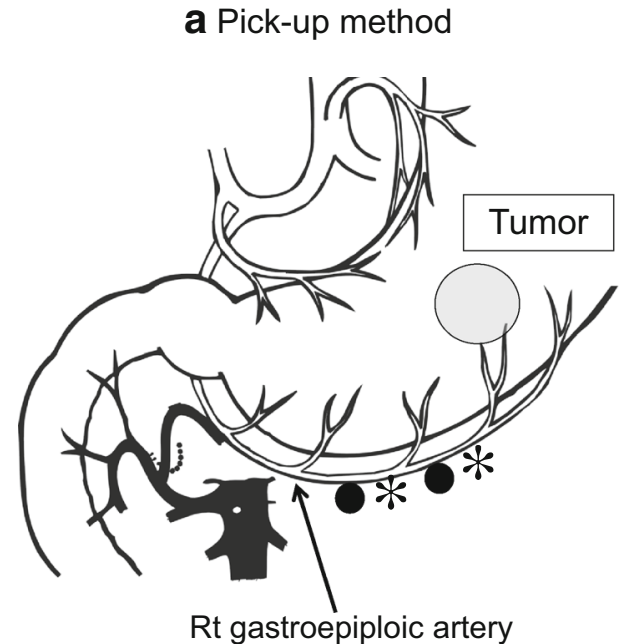

Rt gastroepiploic artery

\section{b Lymphatic basin dissection}

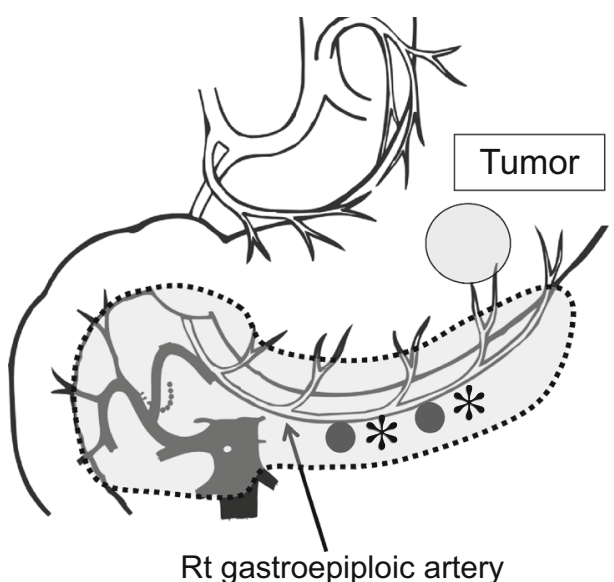


combined technique of complete serial sectioning and immunohistochemistry [23]. Shimizu et al. [28] established a highly sensitive real-time RT-PCR system to detect the messenger RNA (mRNA) of cytokeratin 19, cytokeratin 20, and carcinoembryonic antigen, demonstrating that $27 \%$ of the patients had negative pathological but positive RT-PCR findings. In seven patients $(6.8 \%)$, SNs were negative but non-SNs were positive on RT-PCR in that report. But RT-PCR-positive non-SNs were present in the same station as corresponding SNs in three of these seven patients and in the same basin as SNs in four patients. Furthermore, Kumagai et al. [30] reported that the sensitivity and specificity of the one-step nucleic acid amplification assay compared with histological examination in detecting cytokeratin 19 mRNA were 83.3 and $95.9 \%$, respectively. Recent developments in molecular biological techniques have reduced the detection time to $30-40 \mathrm{~min}$ [31].

These molecular biological methods have dramatically improved the sensitivity of SN metastasis in the intraoperative diagnosis but remain incomplete. It is critical to improve the quality of intraoperative diagnosis in $\mathrm{SN}$ mapping by developing new techniques [32-34]. But basically, allocation bias of metastatic tissue and/or low expression of target mRNA cause false negatives in molecular biological diagnosis. In this sense, the falsenegative rates might not reach $0 \%$ even if there are advancements in molecular biological methods in the future. But almost articles about micrometastasis in SN biopsy support the oncological safety of LBD [23, 28, 35]. LBD is a "de facto standard" technique until a complete intraoperative diagnostic system is established.

\section{Function-preserving gastrectomy as SNNS}

We established function-preserving gastrectomy in combination with LBD on the basis of the favorable results of our feasibility study for the SN concept $[18,36]$. Functionpreserving gastrectomy for early gastric cancer includes not only transectional gastrectomy but also local resection, cardia resection, and antral resection. Similar surgical procedures guided by $\mathrm{SN}$ mapping have been reported [3739].

We show how to perform transectional gastrectomy navigated by SN mapping [36] (Fig. 3). This operation is applied for early gastric cancer of the middle third of the stomach without clinical or radiographic lymph node metastases (cT1N0) and with a diameter less than $4 \mathrm{~cm}$. As mentioned already, SN mapping was conduced by a dual method, using a dye and a radioisotope. ${ }^{99 \mathrm{~m}} \mathrm{Tc}$-phytate or ${ }^{99 \mathrm{~m}}$ Tc-tin colloid is injected into the gastric submucosa at four points around the tumor using an upper

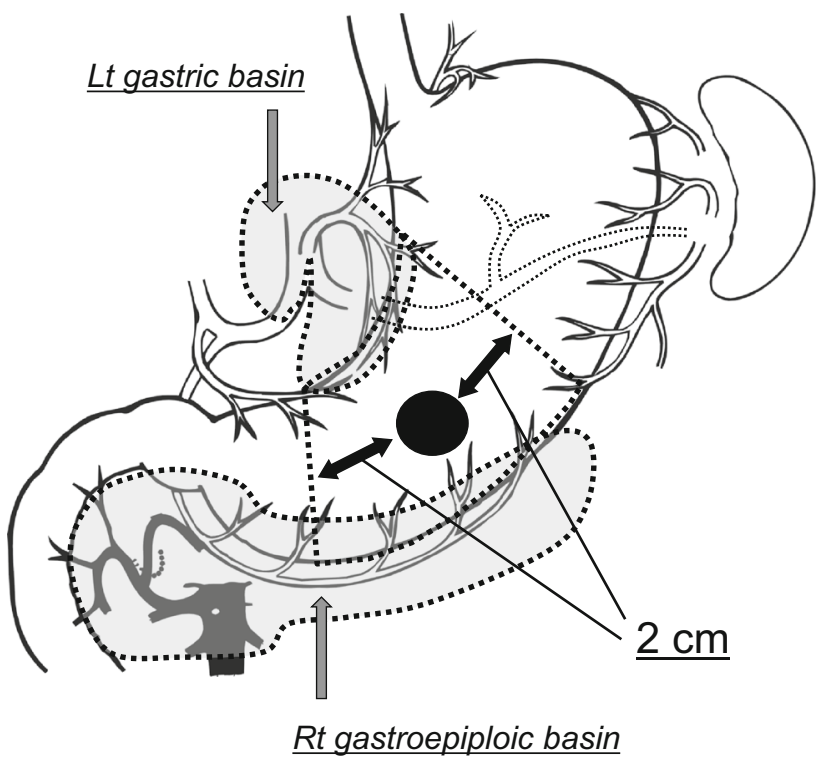

Fig. 3 Transectional gastrectomy is indicated when lymphatic basins along the left gastric artery and the right gastroepiploic artery are stained. After the dissection of both lymphatic basins, stained nodes and hot nodes are retrieved on the back table and are examined intraoperatively. When metastases to sentinel nodes are not detected, transectional gastrectomy is performed. Gastrotomy is necessary to confirm a 2-cm margin from the tumor before transection of the stomach. $L t$ left, $R t$ right

gastrointestinal tract endoscope 1 day before surgery, and sulfan blue or isosulfan blue is injected in the same manner during the operation. About $15 \mathrm{~min}$ after injection of the dye, some lymphatics and lymph nodes are gradually stained blue.

When lymphatic basins along the left gastric artery and the right gastroepiploic artery are stained, transectional gastrectomy is indicated. The LBD, en bloc dissection of the lymphatic basin, is performed and stained nodes and hot nodes are identified by the naked eye and a gamma probe, respectively, on the back table. Retrieved nodes are submitted to the pathology department for intraoperative biopsy. When metastasis is not detected, transectional gastrectomy is performed, but if lymphatic metastases are proven, the standard distal gastrectomy with D2 is selected. Gastrotomy is necessary to confirm a 2-cm margin from the tumor before transection of the stomach. The resected area of the stomach is designed individually depending on the tumor size and location.

It is very important whether function-preserving gastrectomy could improve the patient's postoperative quality of life. There are many studies reporting postoperative objective and subjective outcomes of pylorus-preserving gastrectomy, a subtype of transectional gastrectomy. To summarize, weight loss is less [40], dumping syndrome or early emptying occurs less frequently [41], regurgitant gastritis is milder [42, 43], disturbed bowel movement 
occurs less often [41], function of the gallbladder is more preserved [44], and the incidence of cholecystolithiasis is lower [43] in pylorus-preserving gastrectomy than in conventional distal gastrectomy.

\section{Future perspective}

Although laparoscopy-assisted gastrectomy or laparoscopic gastrectomy is still categorized as clinical research in the gastric cancer treatment guidelines issued by the Japanese Gastric Cancer Association [3], most early gastric cancers are operated on under the laparoscopic approach. The organic combination of low invasiveness by laparoscopic gastrectomy and preservation of gastric function by SNNS will contribute to the patient's short-term and long-term quality of life, respectively [45].

To date, the indication of EMR and ESD for early gastric cancer is limited to histologically differentiated, mucosal tumor with a diameter of less than $2 \mathrm{~cm}$ and without ulcer, whereas the gastric cancer treatment guidelines of the Japanese Gastric Cancer Association suggest some lesions for extended indication in an ongoing clinical trial (JCOG1009/1010) [3]. But these lesions have the possibility of lymph node metastasis. In such a case of risk of lymph node metastasis, endoscopic therapies combined with SN mapping would guarantee oncological security according to the favorable outcome of the multicenter trial conducted by the Japan Society of Sentinel Node Navigation Surgery [7]. When there is no metastasis in SNs as evidenced by intraoperative biopsy, endoscopic therapy would be a sufficient treatment, whereas D2 gastrectomy is necessary if metastases are proved in SNs $[8,46]$. But it is debatable whether endoscopic therapies would be enough to secure a vertical margin. Full-thickness resection is an en bloc procedure dissecting a whole layer of the stomachbearing tumor with selective lymphadenectomy. Then, fullthickness resection is safer than EMR or ESD in oncological radicality because of uncertainty of preoperative diagnosis for tumor invasion and intramural deposits of cancer cells [47] (Fig. 4). Recently, laparoscopic fullthickness resection was developed for gastric tumors. Laparoscopic and endoscopic cooperative surgery is a fullthickness resection for the treatment of submucosal tumor of the stomach [48]. Although laparoscopic and endoscopic cooperative surgery has the merit of minimizing the surgical margin, it induces the exposure of the gastric lesion to the peritoneal cavity, which might lead to iatrogenic implantation and dissemination of cancer cells. More recently, other new techniques such as the combination of laparoscopic and endoscopic approaches to neoplasia with a nonexposure technique (CLEAN-NET) [49] and nonexposed endoscopic wall-inversion surgery (NEWS) $[50,51]$

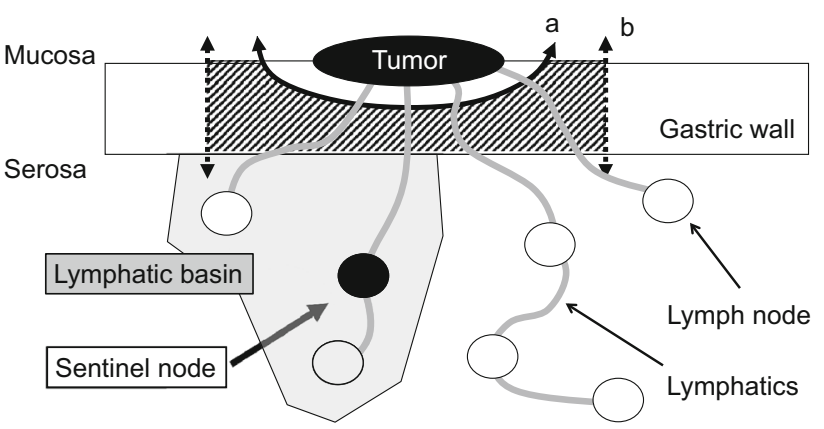

a, Endoscopic therapy ( $)+$ LBD\#

b, Full-thickness resection $(4 \ldots \ldots \ldots . . . \rightarrow)+$ LBD \#

U1IIA Area where cancer cell deposits might be left by ESD in the gastric wall

Fig. 4 It is controversial whether endoscopic therapies such as endoscopic mucosal resection and endoscopic submucosal dissection $(E S D ; a)$ are secure in oncological curability compared with fullthickness resection $(b)$. Because preoperative diagnosis of tumor invasion is uncertain and intramural deposits of cancer cells might be left between the presumed resection lines in the full-thickness resection and the endoscopic therapy, full-thickness resection is preferable at this time. $L B D$ lymphatic basin dissection

have been developed (Fig. 5). After laparoscopic circumferential seromuscular incision, encircled gastric wall with primary tumor is inverted externally in CLEAN-NET or internally in NEWS and resected laparoscopically in CLEAN-NET or endoscopically in NEWS. It is characteristic of CLEAN-NET and NEWS to theoretically protect gastric mucosa against exposure to the peritoneal cavity. These laparoscopic procedures of full-thickness resection in combination with SN mapping are expected to treat early gastric cancer for which EMR or ESD is not applicable.

\section{Conclusions}

SNNS originally aimed at improving the postoperative quality of life of patients by omitting lymphadenectomy in cancer surgery. The pick-up method is an ideal method to minimize postoperative complications. But the importance of metastatic status in SNs is different between superficial malignancies including breast cancer and melanoma, and gastric cancer. Axillar or inguinal lymph node metastasis in the former means systemic disease, whereas regional lymph node metastasis in gastric cancer still has surgical curability. In this sense, the accuracy of metastatic status in SNs must be perfect in gastric cancer. In spite of great developments in SN detection by fluorescence and immunohistochemical or molecular diagnosis, the false-negative rate has not become zero. LBD would be a "de facto standard" technique unless a complete intraoperative diagnostic method for SNs is developed. But further evaluations of the LDB concept and particularly the results of 
Fig. 5 New procedures for early gastric cancer not applicable to endoscopic therapies have been developed. After laparoscopic circumferential seromuscular incision, encircled gastric wall with primary tumor is inverted externally and resected laparoscopically in the combination of laparoscopic and endoscopic approaches to neoplasia with a nonexposure technique (CLEAN-NET; a), whereas encircled gastric wall with primary tumor is inverted internally and resected endoscopically in nonexposed endoscopic wall-inversion surgery $(N E W S ; b)$
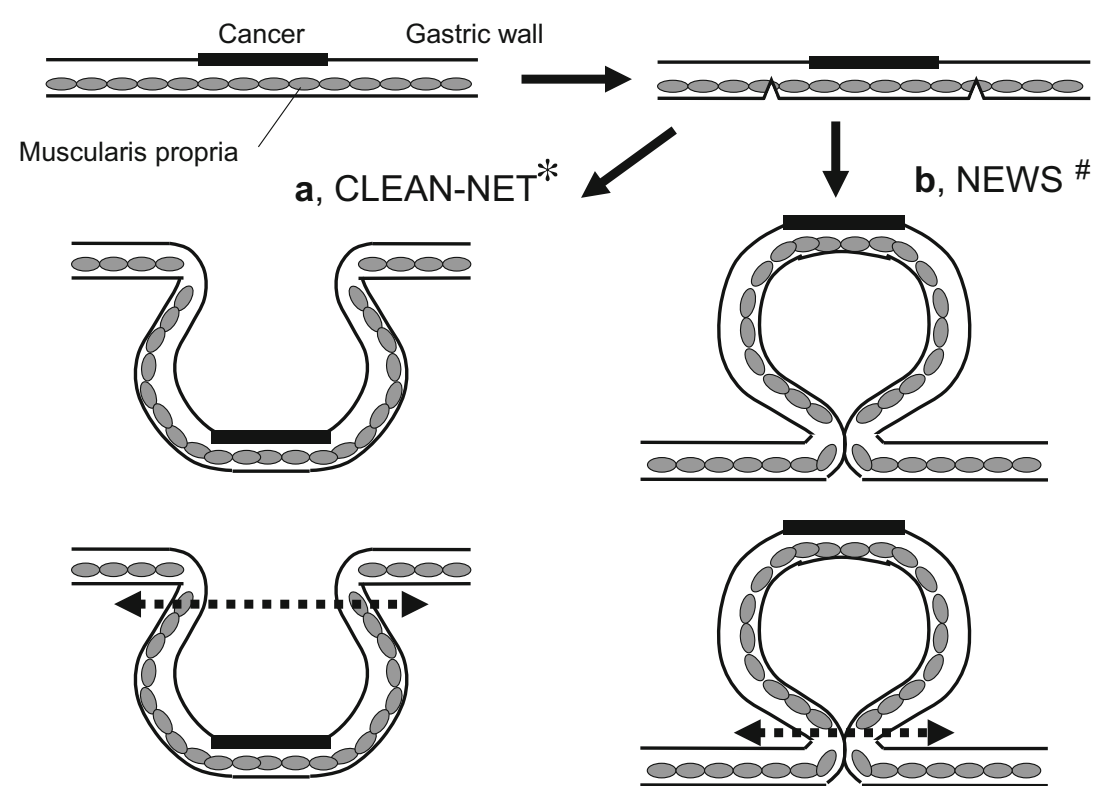

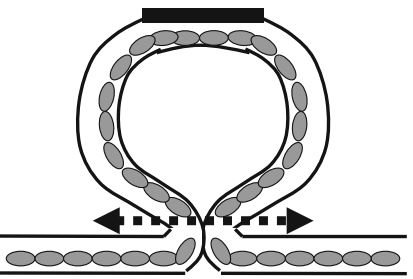

the Japanese trial focused on this topic are needed to elucidate whether the LBD concept is reasonable and function-preserving gastrectomy controlled by LBD would be practical as one of the treatment options for early gastric cancer. Full-thickness resection or ESD in combination with laparoscopic LBD is expected to advance soon. But it is necessary to ameliorate the quality of SN mapping by new techniques and to confirm whether function-preserving gastrectomy could improve postgastrectomy syndrome.

\section{References}

1. Cooperman AM. Postgastrectomy syndromes. Surg Annu. 1981;13:139-61.

2. Eagon JC, Miedema BW, Kelly KA. Postgastrectomy syndromes. Surg Clin North Am. 1992;72:445-65.

3. Japanese Gastric Cancer Association. Japanese gastric cancer treatment guidelines 2010 (ver. 3). Gastric Cancer. 2011;14:113-23.

4. Fujimura T, Kinoshita J, Makino I, Nakamural K, Oyama K, Fujita H, et al. Gastric cancer-state of the art in Japan. Rozhl Chir. 2012;91:346-52.

5. Aikou T, Higashi H, Natsugoe S, Hokita S, Baba M, Tako S. Can sentinel node navigation surgery reduce the extent of lymph node dissection in gastric cancer? Ann Surg Oncol. 2001;8(9 Suppl):90S-3S.

6. Kitagawa Y, Fujii H, Mukai M, Kubo A, Kitajima M. Current status and future prospects of sentinel node navigational surgery for gastrointestinal cancers. Ann Surg Oncol. 2004;11(3 Suppl):242S-4S.

7. Kitagawa Y, Takeuchi H, Takagi Y, Natsugoe S, Terashima M, Murakami N, et al. Sentinel node mapping for gastric cancer: a prospective multicenter trial in Japan. J Clin Oncol. 2013;31:3704-10.

8. Takeuchi H, Kitagawa Y. New sentinel node mapping technologies for early gastric cancer. Ann Surg Oncol. 2013;20:522-32.
9. Ryu KW, Eom BW, Nam BH, Lee JH, Kook MC, Choi IJ, et al. Is the sentinel node biopsy clinically applicable for limited lymphadenectomy and modified gastric resection in gastric cancer? A meta-analysis of feasibility studies. J Surg Oncol. 2011;104:578-84.

10. Wang Z, Dong ZY, Chen JQ, Liu JL. Diagnostic value of sentinel lymph node biopsy in gastric cancer: a meta-analysis. Ann Surg Oncol. 2012;19:1541-50.

11. Miyashiro I, Hiratsuka M, Sasako M, Sano T, Mizusawa J, Nakamura K, et al. High false-negative proportion of intraoperative histological examination as a serious problem for clinical application of sentinel node biopsy for early gastric cancer: final results of the Japan Clinical Oncology Group multicenter trial JCOG0302. Gastric Cancer. 2014;17:316-23.

12. Cox CE, Salud CJ, Cantor A, Bass SS, Peltz ES, Ebert MD, et al. Learning curves for breast cancer sentinel lymph node mapping based on surgical volume analysis. J Am Coll Surg. 2001;193:593-600.

13. Lee JH, Ryu KW, Lee SE, Cho SJ, Lee JY, Kim CG, et al. Learning curve for identification of sentinel lymph node based on a cumulative sum analysis in gastric cancer. Dig Surg. 2010;26:465-70.

14. Nimura H, Narimiya N, Mitsumori N, Yamazaki Y, Yanaga K, Urashima M. Infrared ray electronic endoscopy combined with indocyanine green injection for detection of sentinel nodes of patients with gastric cancer. Br J Surg. 2004;91:575-9.

15. Kelder W, Nimura H, Takahashi N, Mitsumori N, van Dam GM, Yanaga K. Sentinel node mapping with indocyanine green (ICG) and infrared ray detection in early gastric cancer: an accurate method that enables a limited lymphadenectomy. Eur J Surg Oncol. 2010;36:552-8.

16. Kusano M, Tajima Y, Yamazaki K, Kato M, Watanabe M, Miwa M. Sentinel node mapping guided by indocyanine green fluorescence imaging: a new method for sentinel node navigation surgery in gastrointestinal cancer. Dig Surg. 2008;25:103-8.

17. Yoshida M, Kubota K, Kuroda J, Ohta K, Nakamura T, Saito J, et al. Indocyanine green injection for detecting sentinel nodes using color fluorescence camera in the laparoscopy-assisted gastrectomy. J Gastroenterol Hepatol. 2012;Suppl 3:29-33.

18. Miwa K, Kinami S, Taniguchi K, Fushida S, Fujimura T, Nonomura A. Mapping sentinel nodes in patients with early-stage gastric carcinoma. Br J Surg. 2003;90:178-82. 
19. Lee JH, Ryu KW, Kim CG, Kim SK, Choi IJ, Kim YW, et al. Comparative study of the subserosal versus submucosal dye injection method for sentinel node biopsy in gastric cancer. Eur $\mathbf{J}$ Surg Oncol. 2005;31:965-8.

20. Yaguchi Y, Ichikura T, Ono S, Tsujimoto H, Sugasawa H, Sakamoto N, et al. How should tracers be injected to detect for sentinel nodes in gastric cancer-submucosally from inside or subserosally from outside of the stomach? J Exp Clin Cancer Res. 2008;27:79.

21. Kinami S, Fujimura T, Ojima E, Fushida S, Ojima T, Funaki H, et al. PTD classification: proposal for a new classification of gastric cancer location based on physiological lymphatic flow. Int J Clin Oncol. 2008;13:320-9.

22. Ryu KW. The future of sentinel node oriented tailored approach in patients with early gastric cancer. J Gastric Cancer. 2012;12:1-2.

23. Ishii K, Kinami S, Funaki K, Fujita H, Ninomiya I, Fushida S, et al. Detection of sentinel and non-sentinel lymph node micrometastases by complete serial sectioning and immunohistochemical analysis for gastric cancer. J Exp Clin Cancer Res. 2008;27:7.

24. Uenosono Y, Natsugoe S, Ehi K, Arigami T, Hokita S, Aikou T. Detection of sentinel nodes and micrometastases using radioisotope navigation and immunohistochemistry in patients with gastric cancer. Br J Surg. 2005;92:886-9.

25. Ajisaka H, Miwa K. Micrometastases in sentinel nodes of gastric cancer. Br J Cancer. 2003;89:676-80.

26. Takeuchi H, Ueda M, Oyama T, Shimizu Y, Kitagawa Y. Molecular diagnosis and translymphatic chemotherapy targeting sentinel lymph nodes of patients with early gastrointestinal cancers. Digestion. 2010;82:187-91.

27. Arigami T, Natsugoe S, Uenosono Y, Mataki Y, Ehi K, Higashi H, et al. Evaluation of sentinel node concept in gastric cancer based on lymph node micrometastasis determined by reverse transcription-polymerase chain reaction. Ann Surg. 2006;243:341-7.

28. Shimizu Y, Takeuchi H, Sakakura Y, Saikawa Y, Nakahara T, Mukai M, et al. Molecular detection of sentinel node micrometastases in patients with clinical N0 gastric carcinoma with realtime multiplex reverse transcription-polymerase chain reaction assay. Ann Surg Oncol. 2012;19:469-77.

29. Yaguchi Y, Sugasawa H, Tsujimoto H, Takata H, Nakabayashi K, Ichikura $\mathrm{T}$, et al. One-step nucleic acid amplification (OSNA) for the application of sentinel node concept in gastric cancer. Ann Surg Oncol. 2011;18:2289-96.

30. Kumagai K, Yamamoto N, Miyashiro I, Tomita Y, Katai H, Kushima R, et al. Multicenter study evaluating the clinical performance of the OSNA assay for the molecular detection of lymph node metastases in gastric cancer patients. Gastric Cancer. 2014;17:273-80.

31. Yanagita S, Natsugoe S, Uenosono $\mathrm{Y}$, Arigami T, Arima $\mathrm{H}$, Kozono $\mathrm{T}$, et al. Detection of micrometastases in sentinel node navigation surgery for gastric cancer. Surg Oncol. 2008;17:203-10.

32. Soltesz EG, Kim S, Kim SW, Laurence RG, De Grand AM, Parungo CP, et al. Sentinel lymph node mapping of the gastrointestinal tract by using invisible light. Ann Surg Oncol. 2006;13:386-96.

33. Koyama T, Tsubota A, Nariai K, Mitsunaga M, Yanaga K, Takahashi $\mathrm{H}$. Novel biomedical imaging approach for detection of sentinel nodes in an experimental model of gastric cancer. Br J Surg. 2007;94:996-1001.

34. Ojima T, Kinami S, Nakamura K, Oyama K, Inokuchi M, Fujita $\mathrm{H}$, et al. Advantages of the rapid double-staining method for intraoperative detection of micrometastasis in sentinel lymph nodes. Oncol Rep. 2013;30:1067-72.
35. Miyake K, Seshimo A, Kameoka S. Assessment of lymph node micrometastasis in early gastric cancer in relation to sentinel nodes. Gastric Cancer. 2006;9:197-202.

36. Fujimura T, Fushida S, Kayahara M, Ohta T, Kinami S, Miwa K. Transectional gastrectomy: an old but renewed concept for early gastric cancer. Surg Today. 2010;40:398-403.

37. Ichikura T, Sugasawa H, Sakamoto N, Yaguchi Y, Tsujimoto H, Ono S. Limited gastrectomy with dissection of sentinel node stations for early gastric cancer with negative sentinel node biopsy. Ann Surg. 2009;249:942-7.

38. Ohdaira H, Nimura H, Takahashi N, Mitsumori N, Kashiwagi H, Narimiya N, et al. The possibility of performing a limited resection and a lymphadenectomy for proximal gastric carcinoma based on sentinel node navigation. Surg Today. 2009;39:1026-31.

39. Takeuchi H, Oyama T, Kamiya S, Nakamura R, Takahashi T, Wada N, et al. Laparoscopy-assisted proximal gastrectomy with sentinel node mapping for early gastric cancer. World J Surg. 2011;35:2463-71.

40. Isozaki H, Okajima K, Momura E, Ichinona T, Fujii K, Izumi N, et al. Postoperative evaluation of pylorus-preserving gastrectomy for early gastric cancer. Br J Surg. 1996;83:266-9.

41. Nunobe S, Sasako M, Saka M, Fukagawa T, Katai H, Sano T. Symptom evaluation of long-term postoperative outcomes after pylorus-preserving gastrectomy for early gastric cancer. Gastric Cancer. 2007;10:167-72.

42. Nakane Y, Akehira K, Inoue K, Iiyama H, Sato M, Masuya Y, et al. Postoperative evaluation of pylorus-preserving gastrectomy for early gastric cancer. Hepatogastroenterology. 2000;47:590-5.

43. Park DJ, Lee HJ, Jung HC, Kim WH, Lee KU, Yang HK. Clinical outcome of pylorus-preserving gastrectomy in gastric cancer in comparison with conventional distal gastrectomy with Billroth I anastomosis. World J Surg. 2008;32:1029-36.

44. Imada T, Rino Y, Takahashi M, Suzuki M, Tanaka J, Shiozawa $\mathrm{M}$, et al. Postoperative functional evaluation of pylorus-preserving gastrectomy for early gastric cancer compared with conventional distal gastrectomy. Surgery. 1998;123:165-70.

45. Kitagawa Y, Kitano S, Kubota T, Kumai K, Otani Y, Saikawa Y, et al. Minimally invasive surgery for gastric cancer-toward a confluence of two major streams: a review. Gastric Cancer. 2005;8:103-10.

46. Kitagawa Y, Saikawa Y, Takeuchi H, Mukai M, Nakahara T, Kubo A, et al. Sentinel node navigation in early stage gastric cancerupdated data and current status. Scand J Surg. 2006;95:256-9.

47. Takeuchi H, Kitagawa Y. New sentinel node mapping technologies for early gastric cancer. Ann Surg Oncol. 2013;20:522-32.

48. Hiki N, Yamamoto Y, Fukunaga T, Yamaguchi T, Nunobe S, Tokunaga $\mathrm{M}$, et al. Laparoscopic and endoscopic cooperative surgery for gastrointestinal stromal tumor dissection. Surg Endosc. 2008;22:1729-35.

49. Inoue H, Ikeda H, Hosoya T, Yoshida A, Onimaru M, Suzuki M, et al. Endoscopic mucosal resection, endoscopic submucosal dissection, and beyond: full-layer resection for gastric cancer with nonexposure technique (CLEAN-NET). Surg Oncol Clin N Am. 2012;21:129-40.

50. Goto O, Mitsui T, Fujishiro M, Wada I, Shimizu N, Seto Y, Koike K. New method of endoscopic full-thickness resection: a pilot study of non-exposed endoscopic wall-inversion surgery in an ex vivo porcine model. Gastric Cancer. 2011;14:183-7.

51. Goto O, Takeuchi H, Kawakubo H, Matsuda S, Kato F, Sasaki M, et al. Feasibility of non-exposed endoscopic wall-inversion surgery with sentinel node basin dissection as a new surgical method for early gastric cancer: a porcine survival study. Gastric Cancer. 2014. doi:10.1007/s10120-014-0358-y. 